\title{
Tendência da mortalidade relacionada à paracoccidioidomicose, Estado de São Paulo, Brasil, 1985 a 2005: estudo usando causas múltiplas de morte
}

\author{
Augusto Hasiak Santo ${ }^{1}$
}

Como citar

Santo AH. Tendência da mortalidade relacionada à paracoccidioidomicose, Estado de São Paulo, Brasil, 1985 a 2005: estudo usando causas múltiplas de morte. Rev Panam Salud Publica. 2008;23(5):313-24.

RESUMO Objetivo. Estudar a mortalidade relacionada à paracoccidioidomicose informada em qualquer linha ou parte do atestado médico da declaração de óbito.

Métodos. Os dados provieram dos bancos de causas múltiplas de morte da Fundação Sistema Estadual de Análise de Dados (SEADE) de São Paulo entre 1985 e 2005. Foram calculados os coeficientes padronizados de mortalidade relacionada à paracoccidioidomicose como causa básica, como causa associada e pelo total de suas menções.

Resultados. No período de 21 anos ocorreram 1950 óbitos, sendo a paracoccidioidomicose a causa básica de morte em 1164 (59,7\%) e uma causa associada de morte em 786 (40,3\%). Entre 1985 e 2005 observou-se um declínio do coeficiente de mortalidade pela causa básica de 59,8\% e pela causa associada, de 53,0\%. O maior número de óbitos ocorreu entre os homens, nas idades mais avançadas, entre lavradores, com tendência de aumento nos meses de inverno. As principais causas associadas da paracoccidioidomicose como causa básica foram a fibrose pulmonar, as doenças crônicas das vias aéreas inferiores e as pneumonias. As neoplasias malignas e a AIDS foram as principais causas básicas estando a paracoccidioidomicose como causa associada. Verificou-se a necessidade de adequar as tabelas de decisão para o processamento automático de causas de morte nos atestados de óbito com a menção de paracoccidioidomicose.

Conclusões. A metodologia das causas múltiplas de morte, conjugada com a metodologia tradicional da causa básica, abre novas perspectivas para a pesquisa que visa a ampliar o conhecimento sobre a história natural da paracoccidioidomicose.

Palavras-chave Paracoccidioidomicose, causas múltiplas de morte, mortalidade, síndrome de imunodeficiência adquirida, neoplasias, Brasil.

A paracoccidioidomicose é uma micose sistêmica, de natureza granulo-

\footnotetext{
Universidade de São Paulo, Faculdade de Saúde Pública, Departamento de Epidemiologia. Correspondência: Rua Pará 21, apto. 62, CEP 01243-020, São Paulo, SP, Brasil. Fone: +55-11-3258.4760; fax: +55-11-3259.2612. E-mail: auhsanto@usp.br
}

matosa, causada pelo fungo assexuado termo-dimórfico Paracoccidioides brasiliensis. Trata-se de uma micose profunda, geralmente com sintomatologia cutânea importante e grave. Na forma crônica, é conhecida como "tipo adulto" e caracterizada por comprometimento pulmonar, lesões ulceradas de pele, mucosas (oral, nasal, gastrointestinal), baço e fígado e por linfadenopatia. A forma disseminada ou multifocal pode acometer todas as vísceras, sendo freqüentemente afetada a supra-renal. Quando acomete crianças ou adolescentes, apresenta-se na forma mais rara, aguda ou subaguda, 
como "tipo juvenil", comprometendo principalmente o sistema fagocíticomononuclear, atingindo os gânglios linfáticos, o baço e o fígado e levando à disfunção da medula óssea (1-7).

Os casos autóctones da paracoccidioidomicose ocorrem exclusivamente em países da América do Sul e da América Central, em regiões tropicais e subtropicais, particularmente no Brasil, na Colômbia, na Venezuela e na Argentina (1-5). Constitui-se na mais importante micose sistêmica no Brasil, que inclui o maior número de áreas endêmicas no mundo (3), com prevalência proeminente nas regiões Sul, Sudeste e Centro-Oeste, sendo o Estado de São Paulo uma delas (3-7). Em casos graves, a letalidade é estimada entre 2 e $23 \%$, alcançando $30 \%$ se associada à AIDS (3). Pela quantidade de mortes prematuras que provoca, principalmente em segmentos sociais específicos, como os trabalhadores rurais, a paracoccidioidomicose representa um grave problema de saúde pública (2). O coeficiente médio de mortalidade por paracoccidioidomicose como causa básica de morte no Brasil, entre 1980 e 1995, foi de 1,45 óbitos por 1000000 de habitantes. A maior proporção de óbitos nesse período $(29,86 \%)$ ocorreu no Estado de São Paulo (3). No Estado do Paraná, entre 1980 e 1998, esse coeficiente foi de 3,48 óbitos por 1000000 de habitantes $(4,5)$.

As estatísticas de mortalidade têm origem nas declarações de óbito registradas nos cartórios de registro civil, fornecidas por médicos ou instruídas por familiares dos falecidos ou testemunhas $(8,9)$. Os dados demográficos e médicos da declaração de óbito são codificados e processados em serviços de estatística vital ou vigilância epidemiológica nas unidades da federação e enviados ao Ministério da Saúde para a consolidação dos dados do País (10). Dentre os itens da declaração de óbito, destacam-se as causas de morte, das quais aquela que inicia o processo mórbido diretamente responsável pelo êxito letal, denominada causa básica, é aproveitada para a apresentação das estatísticas primárias de mortalidade (11). Em que pese a utilidade desse dado para a prevenção da morte, com o argumento de obter-se uma descrição mais completa de todo o espectro causal, a demanda para a utilização de todas as causas de morte informadas nas declarações de óbito vem crescendo nas últimas décadas $(12,13)$. Essas estatísticas apresentariam, além da causa básica, as demais complicações originadas a partir da causa básica, bem como as outras afecções que tivessem contribuído para determinar a morte. São chamadas de estatísticas pelo método das causas múltiplas de morte $(12,13)$.

Este trabalho objetiva estudar a tendência da mortalidade relacionada à paracoccidioidomicose no Estado de São Paulo por meio da metodologia das causas múltiplas de morte, considerando todas as mortes ocorridas entre 1985 e 2005 em cujas declarações de óbito a paracoccidioidomicose estivesse informada em qualquer linha ou parte do atestado médico da declaração de óbito.

\section{MATERIAIS E MÉTODOS}

Os dados sobre óbitos provieram dos bancos anuais incluindo causas múltiplas de morte da Fundação Sistema Estadual de Análise de Dados (SEADE), responsável pela elaboração das estatísticas vitais em São Paulo. Também foram utilizados os dados sobre a população no período entre 1985 e 2005. Foram estudados todos os óbitos nos quais a paracoccidioidomicose foi mencionada em qualquer linha ou parte do modelo internacional de atestado médico de causa de morte (atestado médico da declaração de óbito) (11), independentemente de ser qualificada como causa básica ou causa associada (não-básica) de morte (12). Foram consideradas conjuntamente como causas associadas as complicações da causa básica (parte I do atestado médico) e as causas contribuintes (parte II do atestado médico) (12).

Os registros dos bancos de óbitos reproduziam em seus campos os respectivos itens da declaração de óbito em uso no Brasil. Neste trabalho foi preciso modificar a estrutura dos registros incluídos nos bancos de dados. Entre 1985 e 2005, vários campos sofreram mudanças em suas designações e tamanhos e nos códigos usados para suas variáveis. Além disso, estiveram em vigor no período duas revisões da Classificação Internacional de Doenças (CID). As características dos campos foram uniformizadas para consolidar os arquivos anuais do período de 21 anos e possibilitar o processamento. Campos auxiliares foram incluídos para facilitar a manipulação dos dados, dentre os quais um campo para transformar em string único os códigos registrados nas linhas (a), (b), (c) e (d), da parte I e da parte II do atestado médico da declaração de óbito.

As causas de morte haviam sido processadas automaticamente entre 1985 e 1995 pelo programa Automated Classification of Medical Entities (ACME) (12, 14, 15), e entre 1996 e 2005 pelo programa Declarações de Óbito de São Paulo (DOSP) (16), que consiste em uma adaptação para processamento em lote do programa Seleção de Causa Básica (SCB) $(14,15)$. Os dados sobre as causas associadas de morte do ano de 1996 são parciais porque a partir do mês de abril daquele ano a Fundação SEADE passou a usar o DOSP. O processamento automático baseia-se em algoritmos e tabelas de decisão que incorporam as regras de mortalidade da Organização Mundial da Saúde (OMS) e as relações etiológicas entre as causas de morte (12-20).

Com a finalidade de reconstituir o processo mórbido que conduziu ao óbito, neste estudo, todas as causas de morte informadas no atestado médico foram consideradas, mesmo as causas mal definidas e aquelas caracterizadas pela OMS como modos de morrer, dentre as quais a "insuficiência respiratória", e a "parada (cardio)respiratória" (11). A paracoccidioidomicose, como causa de morte, correspondeu às formas clínicas incluídas nas subcategorias 116.0 (blastomicose) e 166.1 (paracoccidioidomicose) da Nona Revisão da Classificação Internacional de Doenças (CID-9) e nas categorias B40 (blastomicose) e B41 (paracoccidioidomicose) da Décima Revisão da Classificação Estatística Internacional de 
Doenças e Problemas Relacionados à Saúde (CID-10) (21, 22). Tabelas de equivalência foram preparadas para comparar a tendência das causas de morte relacionadas à paracoccidioidomicose entre essas duas revisões (23, 24). A diferença entre a estrutura de códigos da CID-9, numérica, usada até 1995, e a CID-10, alfanumérica, usada a partir de 1996, determinou o processamento dos dados por meio de programas adaptados a essas estruturas e, por conseqüência, a criação dos seguintes períodos de tempo para o estudo da tendência da mortalidade relacionada à paracoccidioidomicose: 1985 a 1990, 1991 a 1995, 1996 a 2000 e 2001 a 2005. As expressões "óbitos por" ou "devido a" determinada afecção referem-se à causa básica de morte, e as expressões "óbitos com menção de" e "mortalidade relacionada à paracoccidioidomicose" correspondem à presença dessa afecção informada quer como causa básica, quer como associada (não básica), no atestado médico. As causas de morte usadas no estudo foram aquelas mencionadas originalmente no atestado médico (entity axis codes), definidas e apresentadas por meio da estrutura e das rubricas da CID-9 e da CID-10 (12, 20).

Foram estudadas as distribuições das variáveis sexo, idade, idade mediana ao morrer, ocupação, causas básica e associada de morte, número de causas informadas por declaração de óbito e região de residência do falecido, por meio de coeficientes, proporções, sazonalidade, tendência histórica e densidade de óbitos (3) pela área do Estado de São Paulo. As variáveis médicas e demográficas foram processadas pelos programas dBASE III Plus, versão 1.1, Epi Info, versão 6.04d e Excel 2000. O programa Tabulador de Causas Múltiplas (TCM), com versões para a CID-9 e a CID-10 (TCM9, versão 4.0, e TCM-10, versão 2.2), foi usado para a apresentação das causas associadas e do número médio de causas por declaração de óbito (25). Para a apresentação das causas associadas nos óbitos em que a paracoccidioidomicose foi identificada como causa básica, foram preparadas listas especiais que evidenciassem tanto as causas envolvidas na sua história natural quanto as mencionadas com maior freqüência. A duplicação ou multiplicação de causas de morte foi eliminada para sua apresentação por meio de listas abreviadas. Já que o número de causas depende da amplitude da classe (subcategoria, categoria, agrupamento ou capítulo da CID-9 e da CID-10), apenas uma causa foi computada se duas (ou mais) causas mencionadas no atestado médico estivessem incluídas numa mesma classe $(12,25)$. O programa Separador de Registros de Mortalidade (SRM_DBF) (versão 3.1) foi usado para a recuperação de registros em que houvesse interesse em determinadas causas associadas de morte.

Os coeficientes de mortalidade (por 1 000000 de habitantes) foram calculados para os óbitos em que a paracoccidioidomicose foi identificada como causa básica ou associada, bem como para o total de óbitos com suas menções. Para o cálculo dos coeficientes, em cada um dos períodos estudados, o total dos óbitos do período relacionados à paracoccidioidomicose foi dividido pela soma das respectivas populações anuais correspondentes. O Programa para Análise Epidemiológica de Dados Tabulados (Epidat), versão 3.1 (Organização Pan-Americana da Saúde; Xunta de Galícia, Espanha, http://dxsp.sergas.es), foi usado para padronizar por sexo e idade, pelo método direto, os coeficientes de mortalidade no período entre 1985 e 2005 para a população do Brasil em 2000. Os óbitos com sexo ou idade ignorados foram eliminados no cálculo da idade ao morrer e dos coeficientes específicos por idade e sexo. $\mathrm{O}$ indicador da densidade média anual de óbitos (por 10000 quilômetros quadrados) foi calculado para a área do Estado de São Paulo (248 600 quilômetros quadrados).

\section{RESULTADOS}

No Estado de São Paulo, entre 1985 e 2005, ocorreram 1950 óbitos em que a paracoccidioidomicose foi mencionada como causa de morte. Desses, a paracoccidioidomicose foi mencio- nada como causa básica em 1164 $(59,7 \%)$ e como causa associada em 786 $(40,3 \%)$ (tabela 1). Nesse período, o coeficiente médio de mortalidade, incluindo todas as menções da paracoccidioidomicose, foi $67,5 \%$ superior ao coeficiente clássico calculado considerando apenas os óbitos pela causa básica $(2,73$ vs. 1,63 óbitos por 1000000 de habitantes). O número médio foi de 93 óbitos por ano, variando de 128 no ano de 1991 a 73 em 2005, correspondendo, respectivamente, a um coeficiente padronizado de mortalidade pelo total de menções de 4,38 e 1,60 óbitos por 1000000 de habitantes. Observou-se um declínio progressivo dos coeficientes de mortalidade relacionados à paracoccidioidomicose, excetuando-se o aumento verificado nos anos de 1990 e 1991. Esse declínio foi pouco mais acentuado para os coeficientes segundo a causa básica de morte $(59,8 \%)$, variando de 2,28 a 0,92 óbitos por 1000000 de habitantes entre 1985 e 2005. O declínio dos coeficientes segundo a causa associada foi de $53,0 \%$, passando de 1,44 a 0,68 óbitos por 1000000 de habitantes. No início do período, os coeficientes segundo a causa básica eram 58,0\% maiores do que os correspondentes segundo a causa associada de morte, diferença essa que diminuiu progressivamente a ponto de esses coeficientes ficarem iguais no ano de 2002 (figura 1). Ressalve-se que, nesse período de 21 anos, ocorreu uma invasão de 55 óbitos de residentes em outras unidades da federação, os quais, pelo seu pequeno número, foram incluídos neste estudo.

\section{Mortalidade relacionada à paracoccidioidomicose segundo o sexo}

Os coeficientes padronizados de mortalidade relacionada à paracoccidioidomicose, calculados quer pelo total de suas menções, quer considerando a paracoccidioidomicose como causa básica ou causa associada, foram sempre superiores entre os homens. Considerando o período de 1985 a 2005, esses coeficientes, entre os 


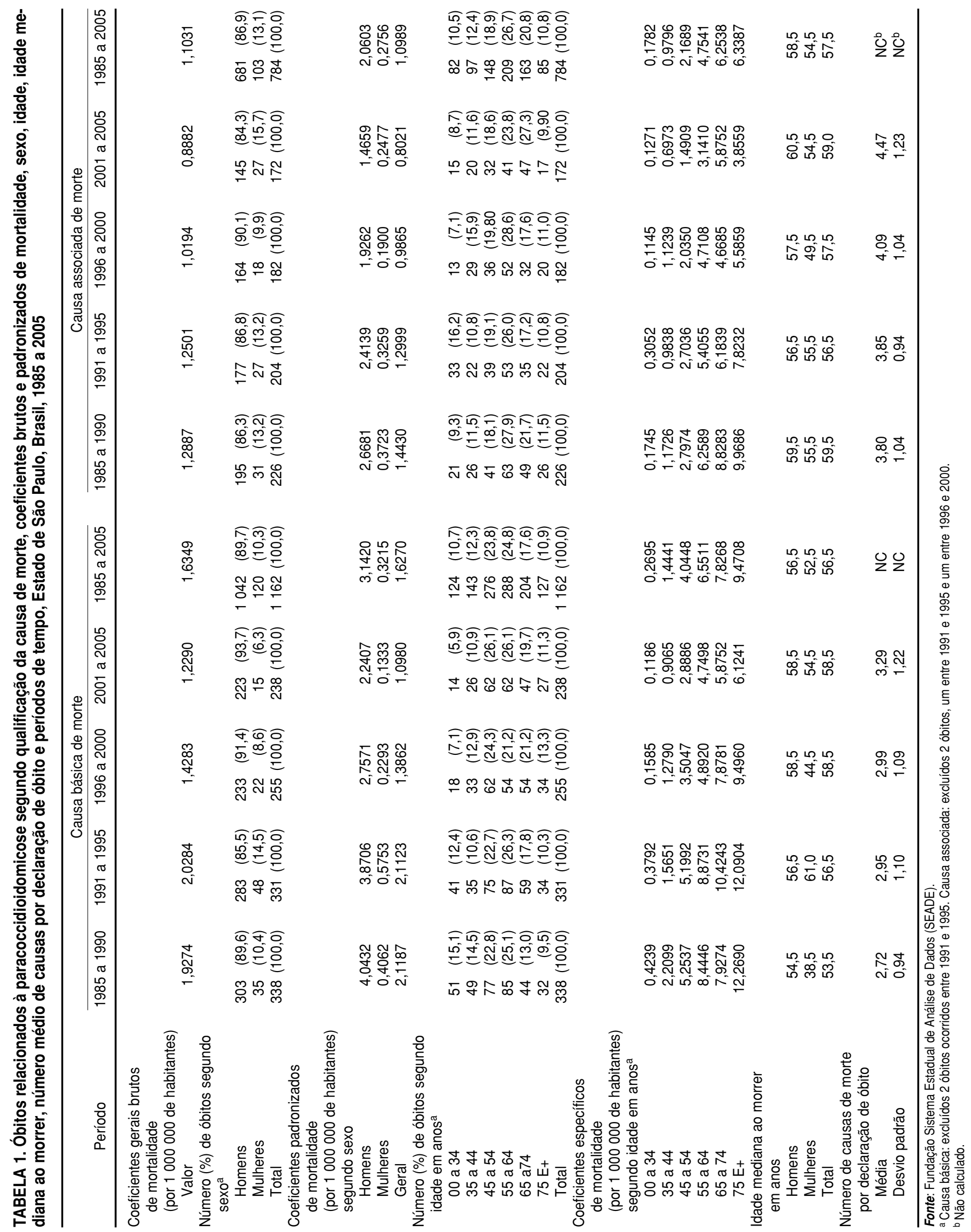


FIGURA 1. Tendência da mortalidade relacionada à paracoccidioidomicose, Estado de São Paulo, Brasil, 1985 a 2005

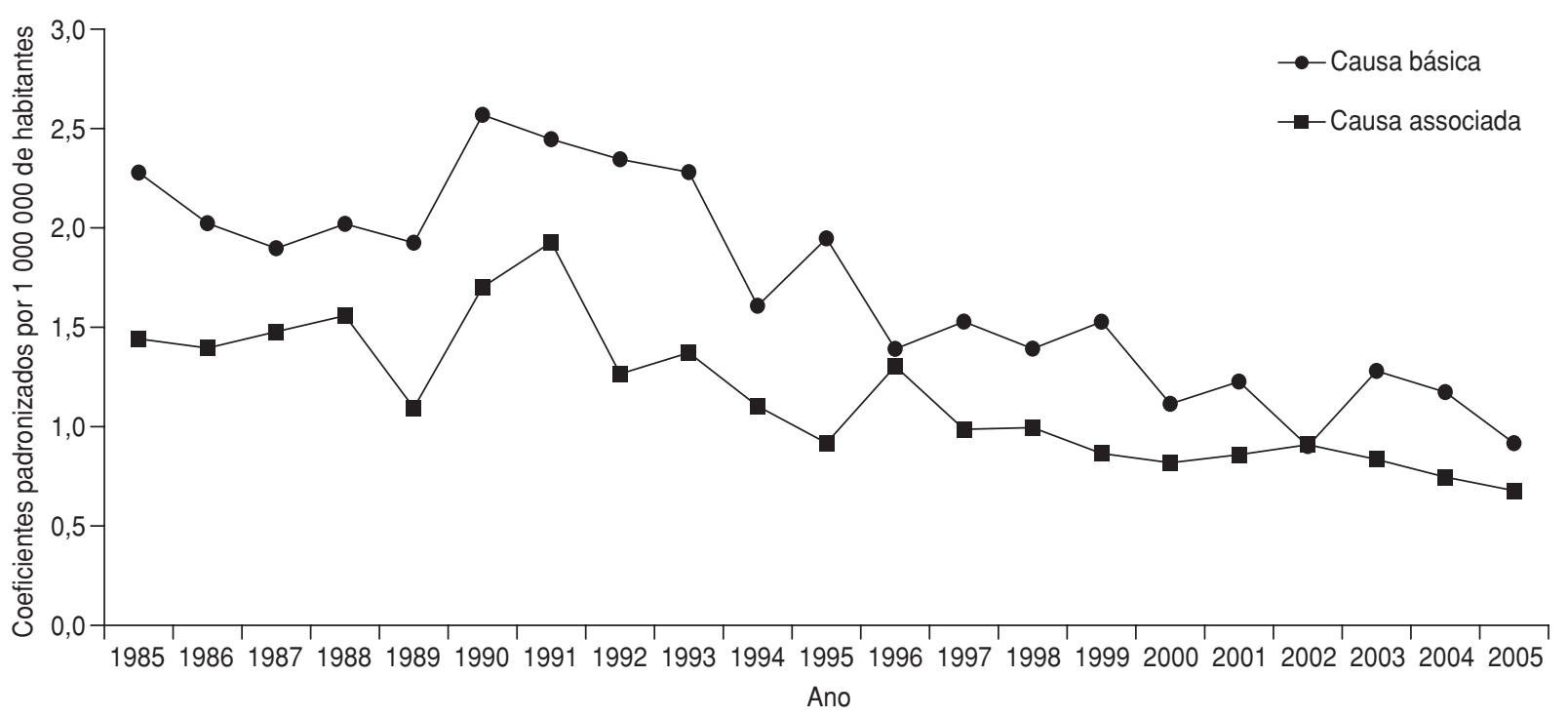

homens, foram, respectivamente, 8,7 (5,20 vs. 0,60 por 1000000 de habitantes), 9,8 e 7,6 vezes maiores do que entre as mulheres (tabela 1). Relativamente à tendência histórica, verificou-se que, apesar de o declínio do coeficiente de mortalidade por paracoccidioidomicose pela causa básica ter sido de $59,0 \%$ entre os homens e de $53,7 \%$ entre as mulheres, a queda dos coeficientes segundo o total de suas menções foi menor entre os homens, de $54,2 \%$, e maior, de $65,5 \%$, na mortalidade das mulheres. Essa diferença deveu-se a um declínio maior do coeficiente de mortalidade segundo a paracoccidioidomicose como causa associada, o qual entre as mulheres foi de $76,9 \%$, em comparação à queda de $45,8 \%$ entre os homens.

\section{Mortalidade relacionada à paracoccidioidomicose segundo a idade}

As maiores proporções de óbitos ocorreram entre os 55 e os 64 anos, com a paracoccidioidomicose como causa básica e principalmente como causa associada de morte. No entanto, os valores dos coeficientes específicos de mortalidade relacionados à paracoccidioidomicose foram diretamente proporcionais às idades dos grupos etários estudados em ambos os sexos, e conseqüentemente os maiores coeficientes foram encontrados no grupo etário de 75 anos ou mais. Esses coeficientes, em praticamente todos os grupos etários considerados, apresentaram valores menores em períodos subseqüentes e declínio geralmente regular e progressivo entre 1985 e 2005; as exceções foram os óbitos pela causa básica nos grupos de 55 a 64 e 65 a 74 anos entre 1985 e 1995 e pela causa associada no grupo de 0 a 34 anos nos subperíodos de 1991 a 1995 e 2000 a 2005, além do grupo de 35 a 44 anos de 1991 a 2000. Os óbitos com as menores idades foram encontrados em dois infantes do sexo masculino, respectivamente de 4 e 10 meses, nos municípios de Águas da Prata em 1987 e Tremembé em 1998, em quem a paracoccidioidomicose se apresentou como causa básica de morte. A idade mais avançada ocorreu em óbito com 95 anos, do sexo masculino, estando a paracoccidioidomicose como causa associada. Vinte e dois óbitos ocorreram em menores de 15 anos de idade (13 homens e nove mulheres), dos quais 18 como causa básica e quatro como causa associada de morte. Para o período total de 21 anos, considerando a paracoccidioidomicose identificada como causa básica, os coeficientes foram respectivamente de 0,27 nos falecidos até 34 anos e de 9,47 óbitos por um 1000000 de habitantes no grupo de 75 anos ou mais. Quando a paracoccidioidomicose se apresentou como causa associada, para os mesmos grupos, esses valores foram, respectivamente, de 0,18 e 6,34 óbitos por 1000000 de habitantes (tabela 1).

\section{Idade mediana ao morrer}

Considerando o período total, a idade mediana ao morrer foi superior entre os homens em relação às mulheres ( 56,5 vs. 52,5 anos como causa básica e 58,5 vs. 54,5 anos como causa associada). Nos óbitos em que a paracoccidioidomicose foi identificada como causa básica, a idade mediana ao morrer foi ligeiramente inferior à idade dos óbitos onde ela foi causa associada de morte (56,5 vs. 57,5 anos). Entre os óbitos por paracoccidioidomicose, ocorreu uma pequena tendência de aumento da idade mediana no período, que passou de 53,5 anos no período de 1985 a 1990 para 58,5 anos entre 2001 e 2005, fato menos evidente entre os óbitos em que a paracoccidioidomicose se apresentou como causa associada (tabela 1). 


\section{Moda e número médio de causas de morte em declarações de óbito}

No decorrer dos quatro subperíodos, observou-se uma tendência de aumento do número médio de causas mencionadas por declaração de óbito. Esse número foi sempre menor para a paracoccidioidomicose como causa básica, respectivamente 2,72, 2,95, 2,99 e 3,29 em relação às respectivas declarações como causa associada $(3,80$, $3,85,4,09$ e 4,47) (tabela 1). Considerando a paracoccidioidomicose como causa básica de morte, a moda para as causas por declaração de óbito foi de 2 entre 1985 e 1990 e de 3 entre 1991 e 2005 , ao passo que, como causa associada ,essa moda foi de 3 e 4 causas no primeiro período e de 4 causas nos três últimos subperíodos estudados.

\section{Designação da paracoccidioidomicose como causa de morte}

Tendo em vista que a designação "paracoccidioidomicose" foi acordada em 1971 na reunião de Medellín (3-5) e o fato de os médicos ainda usarem a designação anterior, "blastomicose", o uso desses termos para a identificação das causas de morte foi avaliado nos óbitos estudados. Como causa básica de morte, o termo "blastomicose" foi usado em 53,8\% dos óbitos e "paracoccidioidomicose" em $46,2 \%$, enquanto que como causa associada esses valores foram respectivamente de $52,6 \%$ e $47,4 \%$. Não foi observado um aumento no uso do termo "paracoccidioidomicose" no decorrer dos quatro subperíodos de tempo nem depois da entrada em vigência da CID-10. No ano de 2002, estando em vigor a CID-10, um atestado de óbito foi preenchido com o uso de ambos os termos, sendo a paracoccidioidomicose indicada como causa associada de morte.

\section{Sazonalidade}

Considerando-se a totalidade dos óbitos ocorridos no período de 21 anos, observou-se uma tendência de au- mento proporcional das mortes relacionadas à paracoccidioidomicose entre os meses de junho e setembro. Entre os homens, esse aumento foi influenciado principalmente pelos óbitos pela causa básica nas idades superiores a 45 anos e pelos óbitos ocorridos nos três primeiros subperíodos entre os anos de 1985 e 2000. A menor influência das mortes entre as mulheres se deveu ao seu número reduzido e à variação proporcional irregular desses óbitos durante os meses do ano.

\section{Causas associadas nas mortes por paracoccidioidomicose}

As causas associadas de morte nos óbitos em que a paracoccidioidomicose foi identificada como causa básica são apresentadas na tabela 2, estando ordenadas pela freqüência decrescente de suas menções no período de 1985 a 1990. Evidenciou-se o predomínio de causas relacionadas ao aparelho respiratório, atuando sob a forma de complicações da paracoccidioidomicose, seguidas das doenças do aparelho circulatório. Destacam-se a fibrose pulmonar nos dois primeiros subperíodos, as doenças crônicas das vias aéreas inferiores que incluem as bronquites, o enfisema e a doença pulmonar obstrutiva crônica, além das pneumonias. Ainda, como causas terminais, destaca-se a menção crescente da insuficiência respiratória como modo de morrer, além da parada (cardio)respiratória e das septicemias. $\mathrm{Na}$ qualidade de causas contribuintes foram mencionadas a desnutrição ou caquexia, a tuberculose e o alcoolismo.

\section{Causas básicas nos óbitos em que a paracoccidioidomicose ocorreu como causa associada de morte}

A tabela 3 mostra as causas básicas de morte nos óbitos em que a paracoccidioidomicose foi informada no atestado médico como causa associada. Prevaleceram as neoplasias malignas, com valores em torno de $15 \%$, ocorrendo em 115 homens e oito mulheres cujas idades medianas foram, respecti- vamente, de 63,5 e 59,0 anos. A doença pelo vírus da imunodeficiência humana teve valores crescentes nos três primeiros períodos entre 1985 e 2000 e decréscimo no último período entre 2001 e 2005. Dentre os 90 óbitos por AIDS, 69 ocorreram em homens e 21 em mulheres, correspondendo à razão de 3,29/1, com idades medianas de, respectivamente, 36,5 e 29,5 anos; a tuberculose foi mencionada como causa associada em $13(14,4 \%)$ desses óbitos. A par dessas causas, verificouse a menção de causas dos aparelhos respiratório e circulatório, que foram também mencionadas como causas associadas nas mortes por paracoccidioidomicose, assinaladas no parágrafo acima.

\section{Distribuição geográfica das mortes relacionadas à paracoccidioidomicose}

A figura 2 mostra os coeficientes brutos de mortalidade relacionada à paracoccidioidomicose segundo as 24 direções regionais de saúde do Estado de São Paulo calculados para o total do período entre 1985 e 2005. Os óbitos de residentes em outras unidades da federação não foram incluídos. As barras da figura são formadas pela soma dos coeficientes pelas causas básica e associada, cujo valores conjuntos resultam no coeficiente pelas menções totais da paracoccidioidomicose nas declarações de óbito. Verifica-se que esses valores para o Estado de São Paulo são, respectivamente, de 1,58, 1,08 e 2,66 óbitos por 1000000 de habitantes. Quinze direções regionais apresentaram coeficientes pelo total de menções de paracoccidioidomicose superiores a 2,66, evidenciando-se os valores de 8,73 em Botucatu, 7,74 em São João da Boa Vista e 7,25 em Barretos. Por outro lado, as direções regionais com maior número de óbitos relacionados à paracoccidioidomicose foram as de Campinas, com 223 óbitos, Capital, com 212, Sorocaba, com 203, Piracicaba, com 119, São José do Rio Preto, com 114, São João da Boa Vista, com 109 e Ribeirão Preto, com 107 óbitos.

No Estado de São Paulo, a densidade da mortalidade segundo as cau- 
TABELA 2. Óbitos por paracoccidioidomicose segundo suas causas associadas de morte, Estado de São Paulo, Brasil, 1985 a $2005^{\mathrm{a}}$

\begin{tabular}{|c|c|c|c|c|c|c|c|c|}
\hline \multirow{2}{*}{$\begin{array}{l}\text { Causas associadas (não-básicas) } \\
\text { de morte (CID-9) (CID-10) }\end{array}$} & \multicolumn{2}{|c|}{$\begin{array}{c}1985 \text { a } 1990 \\
\text { (óbitos }=338 \text { ) }\end{array}$} & \multicolumn{2}{|c|}{$\begin{array}{c}1991 \text { a } 1995 \\
\text { (óbitos = 333) }\end{array}$} & \multicolumn{2}{|c|}{$\begin{array}{r}1996 \text { a } 2000^{c} \\
\text { (óbitos }=255 \text { ) }\end{array}$} & \multicolumn{2}{|c|}{$\begin{array}{l}2001 \text { a } 2005 \\
\text { (óbitos = 238) }\end{array}$} \\
\hline & $n$ & $\%$ & $n$ & $\%$ & $n$ & $\%$ & $n$ & $\%$ \\
\hline Tuberculose (010-018) (A15-A19) & 18 & 5,3 & 14 & 4,2 & 4 & 1,6 & 4 & 1,7 \\
\hline $\begin{array}{l}\text { Septicemias (038) (A40-A41) } \\
\text { Insuficiência adrenocortical }(2554,2558)\end{array}$ & \multicolumn{8}{|c|}{ Insuficiência adrenocortical $(2554,2558)$} \\
\hline $\begin{array}{l}\text { (E271, E274) } \\
\text { Desnutricão/caquexia }\end{array}$ & 10 & 3,0 & 10 & 3,0 & 6 & 2,4 & 11 & 4,6 \\
\hline$(260-263,7994)(E 40-E 46$, R64) & 46 & 13,6 & 47 & 14,1 & 43 & 16,9 & 29 & 12,2 \\
\hline Alcoolismo (303) (F102) & 3 & 0,9 & 15 & 4,5 & 7 & 2,7 & 8 & 3,4 \\
\hline Doenças hipertensivas (401-404) (I10-I13) & 3 & 0,9 & 8 & 2,4 & 3 & 1,2 & 9 & 3,8 \\
\hline \multicolumn{9}{|l|}{ Doenças da circulação pulmonar } \\
\hline \multicolumn{9}{|l|}{$\begin{array}{l}\text { Outras formas de doenças do } \\
\text { coração }(4200-4274,4276-4299)\end{array}$} \\
\hline$(130-\mid 461,147-151)$ & 28 & 8,3 & 24 & 7,2 & 17 & 6,7 & 12 & 5,0 \\
\hline Pneumonias (480-486) (J12-J18) & 34 & 10,1 & 32 & 9,6 & 35 & 13,7 & 56 & 23,5 \\
\hline \multicolumn{9}{|l|}{ Doenças crônicas das vias aéreas } \\
\hline inferiores (490-496) (J40-J47) & 45 & 13,3 & 46 & 13,8 & 37 & 14,5 & 37 & 15,5 \\
\hline Fibrose pulmonar (510-519) (J80-J95, J98) & 89 & 26,3 & 91 & 27,3 & 36 & 14,1 & 25 & 10,5 \\
\hline Insuficiência renal (584-586) (N17-N19) & 8 & 2,4 & 11 & 3,3 & 5 & 2,0 & 17 & 7,1 \\
\hline Choque (7855) (R57) & 14 & 4,1 & 14 & 4,2 & 5 & 2,0 & 2 & 0,8 \\
\hline Insuficiência respiratória (7860) (J96) & 111 & 32,8 & 117 & 35,1 & 153 & 60,0 & 123 & 51,7 \\
\hline \multicolumn{9}{|l|}{ Parada cardiorrespiratória $(4275,7991)$} \\
\hline$(1469, \mathrm{R} 092)$ & 30 & 8,9 & 39 & 11,7 & 33 & 12,9 & 24 & 10,1 \\
\hline \multicolumn{9}{|l|}{ Insuficiência de múltiplos órgãos (7998) } \\
\hline (R688) & 5 & 1,5 & 11 & 3,3 & 11 & 4,3 & 28 & 11,8 \\
\hline Restante das causas associadas & 83 & 24,6 & 87 & 26,1 & 65 & 25,5 & 73 & 30,7 \\
\hline
\end{tabular}

Fonte: Fundação Sistema Estadual de Análise de Dados (SEADE).

a Porcentagens calculadas em relação ao número de óbitos.

${ }^{\text {b }}$ CID 9 = rubricas e códigos da Nona Revisão da Classificação Estatística Internacional de Doenças, Lesões e Causas de Óbito (1985 a 1995). CID 10 = rubricas e códigos da Décima Revisão da Classificação Estatística Internacional de Doenças e Problemas Relacionados à Saúde (1996 a 2004).

c Dados de 1996 parciais.

TABELA 3. Óbitos relacionados à paracoccidioidomicose como causa associada segundo respectivas causas básicas de morte, Estado de São Paulo, Brasil, 1985 a 2005

\begin{tabular}{|c|c|c|c|c|c|c|c|c|}
\hline \multirow{2}{*}{$\begin{array}{l}\text { Causas básicas de morte } \\
\text { (CID-9) }(\text { CID-10) }\end{array}$} & \multicolumn{2}{|c|}{1985 a 1990} & \multicolumn{2}{|c|}{1991 a 1995} & \multicolumn{2}{|c|}{1996 a $2000^{b}$} & \multicolumn{2}{|c|}{2001 a 2005} \\
\hline & $n$ & $\%$ & $n$ & $\%$ & $n$ & $\%$ & $n$ & $\%$ \\
\hline Tuberculose (010-018) (A15-A19) & 13 & 5,8 & 18 & 8,8 & 13 & 7,1 & 7 & 4,1 \\
\hline $\begin{array}{l}\text { Doença pelo vírus da imunodeficiência } \\
\text { humana [HIV] (279.1) (B20-B24) }\end{array}$ & & & & & & & & \\
\hline humana [HIV] (279.1) (B20-B24) & 9 & 4,0 & 26 & 12,7 & 30 & 16,4 & 25 & 14,5 \\
\hline Neoplasias malignas (140-208) (C00-C97) & 36 & 15,9 & 31 & 15,1 & 25 & 13,7 & 31 & 18,0 \\
\hline Doenças isquêmicas do coração & & & & & & & & \\
\hline$(410-414)(120-125)$ & 10 & 4,4 & 15 & 7,3 & 3 & 1,6 & 6 & 3,5 \\
\hline $\begin{array}{l}\text { Doença cardíaca pulmonar e da circulação } \\
\text { pulmonar (415-417) (I26-I28) }\end{array}$ & 21 & 9,3 & 11 & 5,4 & 8 & 4,4 & 4 & 2,3 \\
\hline Outras formas de doenças do coração & & & & & & & & \\
\hline$(390-404,420-429)(100-113, \mid 30-151)$ & 20 & 8,8 & 4 & 2,0 & 17 & 9,3 & 8 & 4,7 \\
\hline Pneumonias (480-486) (J12-J18) & 17 & 7,5 & 13 & 6,3 & 13 & 7,1 & 11 & 6,4 \\
\hline $\begin{array}{l}\text { Doenças crônicas das vias aéreas inferiores } \\
\qquad(490-496)(\mathrm{J} 40-\mathrm{J} 47)\end{array}$ & 17 & 7,5 & 21 & 10,2 & 29 & 15,8 & 30 & 17,4 \\
\hline $\begin{array}{l}\text { Outras doenças do aparelho respiratório } \\
(510-519)(\mathrm{J} 80-\mathrm{J} 95, \mathrm{~J} 98)\end{array}$ & & & 21 & & 11 & & 7 & 41 \\
\hline Restante das causas básicas de morte & 63 & 27,9 & 45 & $\begin{array}{l}10,2 \\
22,0\end{array}$ & 34 & $\begin{array}{r}0,0 \\
18,6\end{array}$ & 43 & $\begin{array}{r}4,1 \\
25,0\end{array}$ \\
\hline Total & 226 & 100,0 & 205 & 100,0 & 183 & 100,0 & 172 & 100,0 \\
\hline
\end{tabular}

Fonte: Fundacão Sistema Estadual de Análise de Dados (SEADE).

${ }^{a}$ CID 9 = rubricas e códigos da Nona Revisão da Classificação Estatística Internacional de Doenças, Lesões e Causas de Óbito (1985 a 1995). CID 10 = rubricas e códigos da Décima Revisão da Classificação Estatística Internacional de Doenças e Problemas Relacionados à Saúde (1996 a 2004).

${ }^{b}$ Dados de 1996 parciais. 
FIGURA 2. Coeficientes brutos de mortalidade (por 1000000 de habitantes) relacionados à paracoccidioidomicose segundo direções regionais de saúde (DIR), Estado de São Paulo, Brasil 1985 a 2005

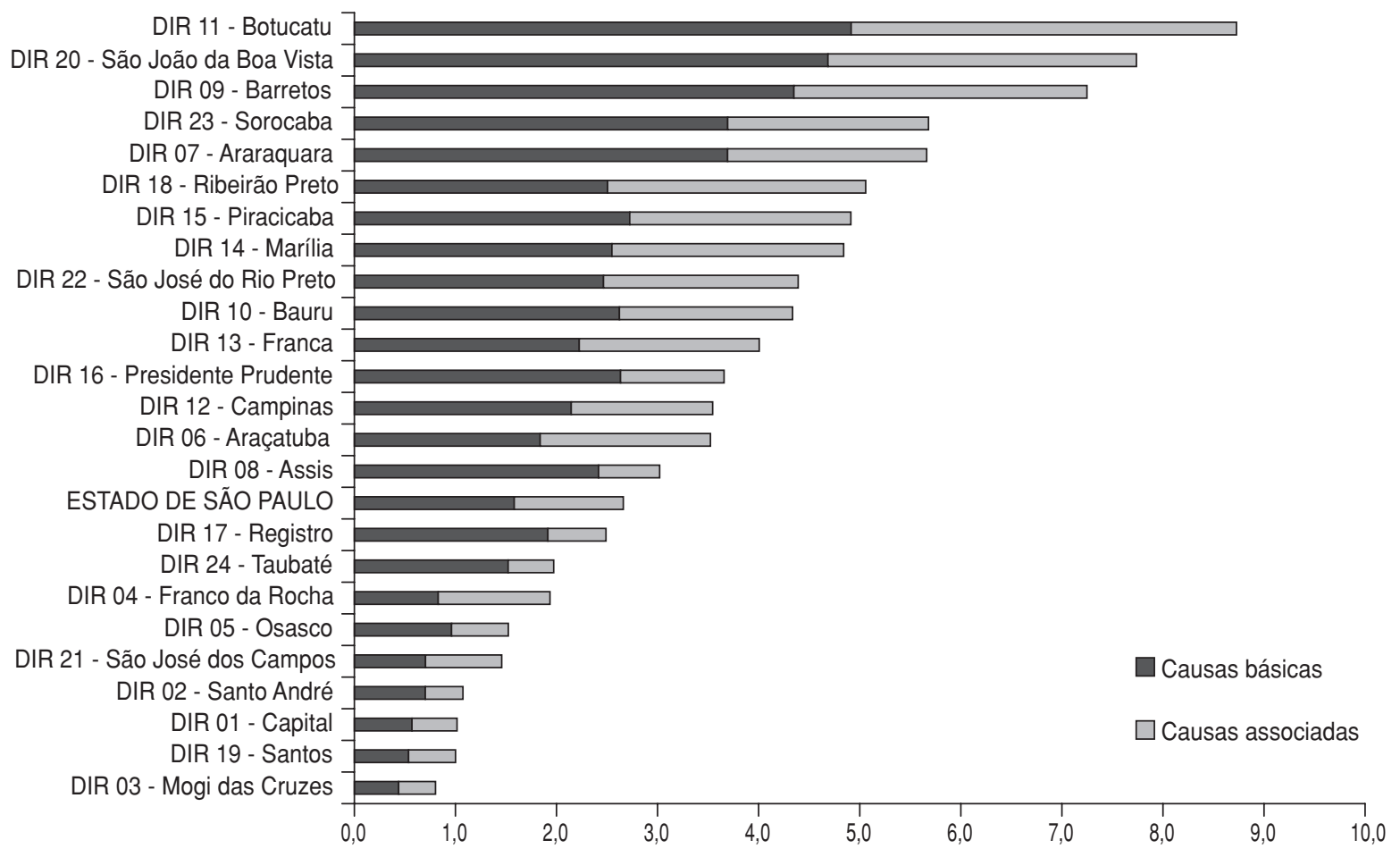

sas básica, associada de morte e total de menções, entre 1985 e 2005, foi, respectivamente, de 2,23, 1,51 e 3,74 óbitos por ano por 10000 quilômetros quadrados.

Observou-se uma variação notável entre os municípios quanto à valorização da paracoccidioidomicose como causa básica de morte nas declarações de óbito. No Estado de São Paulo, para o período entre 1985 e 2005, a paracoccidioidomicose foi identificada como causa básica em 59,4\% das mortes em que foi mencionada (1 124/1 891). No entanto, naqueles municípios em que o número de óbitos relacionados foi superior a 10, a identificação da paracoccidioidomicose como básica variou de 90,9\% (10/11) em Lins até 27,3\% (3/11) em Catanduva. Nas sedes municipais das direções regionais com maior número de óbitos mencionadas no parágrafo anterior, essa proporção foi de 56,6\% em Campinas, 56,1\% na Capital, 67,3\% em Sorocaba, 50,0\% em Piracicaba, 40,9\% em São José do Rio
Preto, $62,5 \%$ em São João da Boa Vista e 38,0\% em Ribeirão Preto.

\section{Ocupação}

O estudo da ocupação foi realizado considerando-se o total de óbitos relacionados à paracoccidioidomicose e eliminando-se os registros de falecidos que no momento da morte estavam fora do mercado de trabalho (desocupados, inválidos, aposentados, prendas domésticas e estudantes, entre outros) e de ocupação não classificada. Dentre os 1935 registros em que o campo sobre ocupação estava preenchido nas declarações de óbito, 875 $(45,2 \%)$ incluíam dados sobre o tipo de trabalho desenvolvido pelo falecido. Nesses óbitos, as ocupações prevalentes foram as de trabalhador agropecuário, florestal, da pesca e trabalhadores assemelhados, em número de 330 $(37,7 \%), 107(12,2 \%)$ trabalhadores da construção civil e assemelhados e 80
$(9,1 \%)$ condutores de veículos de transporte e trabalhadores assemelhados.

\section{DISCUSSÃO}

O uso de todas as causas mencionadas no atestado médico das declarações de óbito permitiu evidenciar que a responsabilidade da paracoccidioidomicose na determinação das mortes teve sua importância acrescida em $67,5 \%$ em comparação aos coeficientes clássicos calculados apenas pela causa básica. Não sendo a paracoccidioidomicose uma entidade de notificação compulsória, as suas menções como causa de morte, por meio de medidas de vigilância epidemiológica, podem ser usadas para medir o seu impacto sobre a saúde da população (3).

Observou-se o declínio dos coeficientes de mortalidade relacionada à paracoccidioidomicose como causa básica e associada no período entre 
1985 e 2005. De modo particular para a causa básica de morte, esse declínio pode ser referendado pela comparação entre o coeficiente médio padronizado desse período e os coeficientes médios verificados para os períodos de 1980 a 1995 e de 1980 a 1998 em estudos anteriores $(3,4)$, respectivamente de 1,62, 2,02 e 1,91 óbitos por 1000000 de habitantes, bem como pelos correspondentes números médios de óbitos por ano, respectivamente de 55,43 e 59,38 e 57,84 .

De modo consistente com outros estudos, a mortalidade relacionada à paracoccidioidomicose foi maior entre os homens. No Estado de São Paulo entre 1985 e 2005, entretanto, pela causa básica, o coeficiente de mortalidade padronizado de 3,14 foi menor do que o encontrado para o Estado do Paraná (4) entre 1980 e 1995, de 5,91 óbitos por 1000000 de habitantes, fato que pode ser atribuído à tendência de queda mencionada acima. Por outro lado, a queda maior da mortalidade entre as mulheres nos óbitos em que a paracoccidioidomicose apresentou-se como causa associada pode estar relacionada à epidemia de AIDS atingir principalmente os homens, o que poderia ter determinado também o declínio menos acentuado do coeficiente de mortalidade pela causa associada de morte em relação àquele calculado segundo a causa básica.

$\mathrm{O}$ fato de os valores dos coeficientes de mortalidade serem praticamente proporcionais às idades dos falecidos e à concentração dos óbitos nas idades mais avançadas refletem o caráter de cronicidade da paracoccidioidomicose. Embora a infecção possa ter ocorrido em idades mais precoces, a doença poderá manifestar-se somente na idade adulta. Corrobora esse argumento a idade mediana geral ao morrer, que foi superior a 55 anos. Neste trabalho, foram observados dois óbitos de infantes, que acreditamos corresponder aos casos de paracoccidioidomicose com as idades menores já registrados na literatura.

As proporções de uso dos termos paracoccidioidomicose e blastomicose para descrever as causas básicas de morte no Estado de São Paulo (46,22 e
$53,78 \%$ ) aproximaram-se daquelas que foram encontradas no estudo do Estado do Paraná $(43,74$ e 56,26\%) (4) e diferiram das verificadas no Brasil (54,04 e 45,96\%) (3). O uso dessas designações está relacionado com a formação dos médicos que preenchem as declarações de óbito $(4,12,20)$. No entanto, com os dados atuais, torna-se difícil levantar hipóteses explicativas precisas sobre essas diferenças, sendo necessário o estudo dessa distribuição em outras unidades da federação.

$\mathrm{O}$ achado de uma maior proporção de óbitos entre os meses de junho e setembro nas idades superiores a 45 anos pode estar relacionado ao comprometimento pulmonar determinado pela paracoccidioidomicose. $\mathrm{O}$ aumento da morbidade e da mortalidade devidas às doenças respiratórias nos meses de inverno (26-28), que atinge principalmente os idosos, tem sido observado em nosso meio (29). As manifestações pulmonares ocorrem em $90 \%$ dos pacientes com a forma crônica da micose, tendo sido descrita fibrose pulmonar por imagem em cerca de 50 \% de pacientes com infecção crônica (2). Neste estudo, a fibrose pulmonar foi a principal causa de morte relacionada à paracoccidioidomicose.

O cômputo de todas as causas informadas nos atestados médicos enseja a reconstituição do processo mórbido determinante da morte pela paracoccidioidomicose. O conhecimento das causas associadas aporta subsídios importantes para evitar a morte. A prevenção e o tratamento das complicações da paracoccidioidomicose, bem como de outras afecções contribuintes, detêm o processo letal e aumentam a sobrevida de doentes. De modo consistente com as descrições clínicas, as complicações pulmonares destacaramse como principais causas associadas, particularmente a fibrose pulmonar e a doença pulmonar obstrutiva crônica, agravadas pelas pneumonias. Do mesmo modo, ficou evidente a importância da desnutrição ou da caquexia como causa contribuinte da morte, além do alcoolismo e da tuberculose, cuja importância como fator de risco da paracoccidioidomicose tem preocupado os pesquisadores $(7,30,31)$.
A recuperação dos óbitos em que a paracoccidioidomicose se apresentou como causa associada constitui-se em outra das propriedades da metodologia das causas múltiplas. A identificação das causas básicas responsáveis por esses óbitos amplia o conhecimento epidemiológico da mortalidade relacionada à paracoccidioidomicose. Neste estudo, os óbitos devidos às neoplasias malignas prevaleceram. Existem evidências de que a terapia antineoplásica favorece a reativação da paracoccidioidomicose e de que os linfomas e as leucemias estão relacionados às formas clínicas mais graves da micose, estando a elevada letalidade principalmente relacionada às neoplasias (32). Por outro lado, os carcinomas teriam localizações atingidas previamente pela paracoccidioidomicose, geralmente na forma crônica, com atividades evolutivas paralelas (32). Estudos anteriores verificaram a importância das neoplasias como causa básica na determinação das mortes relacionadas à paracoccidioidomicose $(7,33)$.

A necessidade de considerar-se a co-infecção entre a paracoccidioidomicose e a AIDS tem sido enfatizada desde o primeiro relato dessa associação $(32,34)$. Um estudo sobre a avaliação dos efeitos da infecção pelo HIV na história natural da paracoccidioidomicose mostrou que as características clínicas desses pacientes assemelhamse pela gravidade às características encontradas na forma aguda, e que a doença resulta da reativação a partir da infecção latente nos hospedeiros imunocomprometidos pelo HIV (35). Nesse estudo, a tuberculose foi uma das infecções oportunistas mais freqüentes, consistente com os achados entre os óbitos do Estado de São Paulo (35). A disseminação da epidemia da AIDS para as áreas rurais do Brasil faz prever um aumento da sua associação com a paracoccidioidomicose, motivo da recomendação para a inclusão da paracoccidioidomicose dentre as afetadas pela AIDS (36).

Outra importante contribuição procedente da metodologia das causas múltiplas refere-se à avaliação do processamento automático para a identifi- 
cação da causa básica de morte. Neste estudo, várias complicações respiratórias e circulatórias da paracoccidioidomicose foram identificadas como causa básica de morte. O processamento automático usa tabelas de decisão para interpretar a relação causal entre as afecções mencionadas no atestado médico. Entretanto, essas tabelas, preparadas pelo National Center for $\mathrm{He}$ alth Statistics, dos Estados Unidos (18, 19), embora aceitas internacionalmente, não prevêem essa relação entre causas raras $(37,38)$. A propósito, cabe lembrar que, para a introdução no Estado de São Paulo, no ano de 1983, houve necessidade de adaptar as tabelas de decisão integrantes do sistema ACME para o processamento de mortes relacionadas à doença de Chagas e à esquistossomose. Neste estudo, as causas de morte identificadas originalmente foram aceitas sem controle suplementar. Conseqüentemente, a causa básica de morte poderia ser a paracoccidioidomicose nos óbitos em que como tal foram identificadas as doenças crônicas das vias aéreas inferiores, as pneumonias, as doenças cardíacas pulmonares e da circulação pulmonar e outras doenças do aparelho respiratório.

A distribuição dos óbitos segundo as direções regionais de saúde é consistente com os relatos sobre as áreas endêmicas da paracoccidioidomicose no Estado de São Paulo. Embora o estudo dos óbitos tenha sido feito pelo local de residência, cabe uma ressalva quanto à possível invasão de óbitos em algumas dessas regiões, particularmente aquelas que contam com serviços de referência procurados pelos doentes para receber diagnóstico e tratamento $(6,7)$. Neste estudo, houve 55 óbitos de residentes em outras unidades da federação. Os maiores coeficientes de mortalidade foram verificados na regional de Botucatu, em que 20,2\% (45/223) dos óbitos ocorreram nos municípios da sede, São Manuel e Avaré, coincidente com a distribuição de doentes (6). Por outro lado, considerando a paracoccidioidomicose como causa básica de morte, verificouse a queda da densidade anual de óbitos por 10000 quilômetros quadrados no Estado de São Paulo, de 2,39 no período de 1980 a 1995 (3) para 2,23 entre 1985 e 2005.

Igualmente consistente com as observações encontradas na literatura, as ocupações mais freqüentes foram as de trabalhador em atividades agrícolas, da construção civil $(3,4,6,7)$ e motorista. Admite-se que o solo seja o reservatório do Paracoccidioides brasiliensis na natureza, a partir de onde, por inalação, instala-se a infecção nos lavradores. As profissões ou as atividades relacionadas ao manejo do solo contaminado com o fungo são os principais fatores de risco da infecção (2). No Brasil, a exposição ocupacional ao Paracoccidioides brasiliensis, principalmente em trabalhos agrícolas ou florestais e em zonas endêmicas, é considerada uma doença profissional (39).

Este estudo apresenta as limitações inerentes às estatísticas de mortalidade (12). Dentre as limitações quantitativas, o sub-registro de óbitos mostra-se irrelevante no Estado de São Paulo. As estimativas entre os óbitos informados à Fundação SEADE e os estimados por meio de projeções demográficas, disponíveis desde 1991, mostram que, para o Estado de São Paulo, os valores superam os $100 \%$, indicando boa cobertura dos dados sobre mortalidade na população (40). As limitações qualitativas referem-se à confiabilidade dos dados demográficos e médicos, em especial sobre as causas de morte. A qualidade desses dados depende, em grande parte, do conhecimento por parte dos médicos atestantes da importância da declaração de óbito como fonte das estatísticas de mortalidade $(9,12)$. $\mathrm{O}$ indicador mais usado para avaliar a qualidade desse dados consiste na proporção de causas mal definidas. No Estado de São Paulo, como causa básica de morte, as causas mal definidas atingiram, respectivamente, 6,0, 6,3, 6,4 e $6,5 \%$ dentre o total de óbitos em cada um dos quatro períodos estudados, valores inferiores à metade dos correspondentes para o País. Verifica-se que o trabalho do codificador das causas de morte é acurado e não se constitui em fonte de erro $(17,37)$. A propósito, no Estado do Paraná, os codificadores tomaram de modo adequado a iniciativa de usar códigos reservados para a paracoccidioidomicose quando, no atestado médico, a blastomicose estava mencionada como causa de morte $(4,5)$. Um comentário anterior discutiu a falta de previsão de tabelas de decisão para o processamento automático da paracoccidioidomicose como causa de morte. Neste estudo, essa limitação foi contornada por meio da metodologia das causas múltiplas, considerando a paracoccidioidomicose independentemente de ter sido qualificada como causa básica ou associada de morte.

Um tópico importante relativo à qualidade dos dados refere-se à subestimação de mortes relacionadas à paracoccidioidomicose. Verificou-se que, no Estado de São Paulo, a paracoccidioidomicose foi identificada como causa básica em 59,4\% das declarações de óbito em que estava mencionada, proporção que variou de 90,9 a $27,3 \%$ nos municípios com mais de 10 mortes e de 56,6 a $38,0 \%$ nas sedes municipais das direções regionais de saúde. Essas diferenças resultam da valorização atribuída à paracoccidioidomicose como causa básica pelos médicos responsáveis pelo fornecimento da declaração de óbito. Vários fatores influenciam essa valorização, dentre outros a formação médica recebida e a especialidade exercida, a disponibilidade de meios e estrutura para o diagnóstico, o conhecimento sobre a importância médica e legal e sobre as diretrizes para o fornecimento da declaração de óbito e a coexistência de outras causas de morte $(12,20)$. Além disso, a subestimação pode ocorrer pela falta do registro de determinadas causas de morte. Afirma-se que esse fato ocorre com grande probabilidade quando da associação entre a AIDS e a paracoccidioidomicose (41). Essa eventualidade foi verificada em um estudo sobre a resistência do Mycobacterium tuberculosis às drogas em pacientes HIV-positivos, em que, dentre as 99 mortes ocorridas, a tuberculose estava mencionada apenas em 49 (49,5\%) das declarações de óbito $(42,43)$.

O presente estudo descreveu as características da mortalidade relacio- 
nada à paracoccidioidomicose identificada por meio da metodologia das causas múltiplas de morte. A consideração dos óbitos em que a paracoccidioidomicose ocorreu como causa associada acresceu em $67,5 \%$ o coeficiente de mortalidade calculado tradicionalmente. No estudo, e em comparação com trabalhos anteriores, ficou evidente o declínio da mortalidade re- lacionada à paracoccidioidomicose, além de terem sido reproduzidos dados sobre fatores de risco ligados ao sexo, à idade e à ocupação. Os óbitos em infantes de 4 e 10 meses de idade são os mais precoces registrados na literatura. $\mathrm{O}$ aumento verificado das mortes nos meses de inverno, a proeminência das complicações respiratórias associadas, bem como a causali- dade adjunta das neoplasias malignas e da AIDS contribuem para orientar o controle da mortalidade relacionada à paracoccidioidomicose. $\mathrm{O}$ uso da metodologia das causas múltiplas de morte, conjugado com a tradicional da causa básica, abre novas perspectivas para a pesquisa visando a ampliar o conhecimento sobre a história natural da paracoccidioidomicose.

\section{REFERÊNCIAS}

1. Wanke B, Londero AT. Epidemiology and paracoccidioidomycosis infection. Em: Franco M, Lacaz CS, Restrepo-Moreno A, Del Negro G, eds. Paracoccidioidomycosis. Boca Raton, Florida: CRC Press; 1993. Pp. 109-20.

2. Shikanai-Yasuda MA, Telles Filho FQ, Mendes RP, Colombo AL, Moretti ML; Grupo de Consultores do Consenso em Paracoccidioidomicose. Consenso em paracoccidioidomicose. Rev Soc Bras Med Trop. 2006;39(3): 297-310.

3. Coutinho ZF, Silva D, Lazéra M, Petri V, Oliveira RM, Sabroza PC, et al. Paracoccidioidomycosis mortality in Brazil. Cad Saude Publica. 2002;18(5):1441-54.

4. Bittencourt JIM, Oliveira RM, Coutinho ZF. Paracoccidioidomycosis mortality in the State of Paraná, Brazil, 1980/1998. Cad Saude Publica. 2005;21(6):1856-64.

5. Bittencourt JIM. Mortalidade por paracoccidioidomicose no Estado do Paraná, Brasil (1980-1998) [dissertação de mestrado]. Ponta Grossa: Universidade Estadual de Ponta Grossa; 2002.

6. Marques SA, Franco MF, Mendes RP, Silva NCA, Baccili C, Curcelli ED, et al. Aspectos epidemiológicos da paracoccidioidomicose na área endêmica de Botucatu (São PauloBrasil). Rev Inst Med Trop Sao Paulo. 1983; 25(2):87-92.

7. Blotta MHSL, Mamoni RL, Oliveira SJ, Nouér SA, Papajordanou PMO, Gouveia A, et al. Endemic regions of paracoccidioidomycosis in Brazil: a clinical and epidemiologic study of 584 cases in the Southeast Region. Am J Trop Med Hyg. 1999;61(3):390-4.

8. Brasil. Lei n. 6.015 , de 31 de dezembro de 1973. Dispõe sobre os registros públicos, e dá outras providências. Diário Oficial da União 1973; 31 dez. Disponível em http://www. planalto.gov.br/CCIVIL/LEIS/L6015.htm. Acessado em 7 de dezembro de 2007.

9. Conselho Federal de Medicina. Resolução CFM n ${ }^{\circ} 1779 / 2005$. Regulamenta a responsabilidade médica no fornecimento da Declaração de Óbito. Diário Oficial da União 2005; 5 dez: seção I, p. 121. Disponível em http:// www.portalmedico.org.br/resolucoes/cfm/ 2005/1779_2005.htm. Acessado em 7 de agosto de 2007.

10. Brasil, Ministério da Saúde, Fundação Nacional da Saúde. Manual de Procedimentos do Sistema de Informações sobre Mortali- dade. Brasília: Ministério da Saúde/Fundação Nacional de Saúde; 2001.

11. Organização Mundial da Saúde. Classificação estatística internacional de doenças e problemas relacionados à saúde: décima revisão. Vol. 2. Manual de instrução. São Paulo: Centro Colaborador da OMS para a Classificação de Doenças em Português/Edusp; 1993.

12. Santo AH. Causas múltiplas de morte: formas de apresentação e métodos de análise [tese de doutorado]. São Paulo: Faculdade de Saúde Pública, Universidade de São Paulo; 1988.

13. Israel RA, Rosenberg HM, Curtin LR. Analytical potential for multiple cause-of-death data. Am J Epidemiol. 1986;124(2):161-79.

14. Santo AH, Pinheiro CE. Uso do microcomputador na seleção da causa básica de morte. Bol Oficina Sanit Panam. 1995;119(4):319-27.

15. Santo AH, Pinheiro CE, Rodrigues EM. Comparative evaluation of underlying causes of death processed by the Automated Classification of Medical Entities and the Underlying Cause of Death Selection Systems. Rev Saude Publica. 1998;32(1):1-6.

16. Pinheiro CE, Santo AH. Processamento de causas de morte em lote pelo sistema de seleção de causa básica. Rev Saude Publica. 1998;32(1):72-3.

17. Santo AH. Avaliação da qualidade da codificação das causas de morte no Estado de São Paulo, Brasil. IESUS. 2000;9(3):189-98.

18. National Center for Health Statistics. Instruction Manual 2c: ICD-9 ACME decision tables for classifying the underlying causes of death, 1992. Hyattsville, Maryland: NCHS; 1992.

19. National Center for Health Statistics. Instruction Manual 2c: ICD-10 ACME decision tables for classifying the underlying causes of death, 2006. Hyattsville, Maryland: NCHS; 2005.

20. Wall MM, Huang J, Oswald J, McCullen D. Factors associated with reporting multiple causes of death. BMC Med Res Methodol. 2005;5(1):4.

21. Organização Mundial da Saúde. Manual da classificação estatística internacional de doenças, lesões e causas de óbito: nona revisão. Vol. 1. São Paulo: Centro da OMS para a Classificação de Doenças em Português/ MS/USP/OPAS; 1985.

22. Organização Mundial da Saúde. Classificação estatística internacional de doenças e problemas relacionados à saúde: décima revisão. Vol. 2. Manual de instrução. São Paulo: Cen- tro Colaborador da OMS para a Classificação de Doenças em Português/Edusp; 1993.

23. Santo AH. Equivalência entre revisões da Classificação Internacional de Doenças: causas de morte. Rev Saude Publica. 2000; 34(1):21-8.

24. World Health Organization. International Classification of Diseases Translator: ninth and tenth revisions. Genebra: WHO; 1997.

25. Santo AH, Pinheiro CE. Tabulador de causas múltiplas de morte. Rev Brasil Epidemiol. 1999;2(1/2):90-7.

26. Victora CG, Vaughan JP, Barros FC. The seazonality of infant deaths due to diarrheal and respiratory diseases in southern Brazil, 19741978. Bull Pan Am Health Organ. 1985;19(1): 29-39.

27. Toyoshima MTK, Ito GM, Gouveia N. Tendências temporais da morbidade e da mortalidade por doenças respiratórias na cidade de São Paulo. Rev Med (São Paulo). 2003;82(1-4):67-77.

28. Toyoshima MTK, Ito GM, Gouveia N. Morbidade por doenças respiratórias em pacientes hospitalizados em São Paulo/SP. Rev Assoc Med Bras. 2005;51(4):209-13.

29. Francisco PMSB, Donalisio MRC, Latorre MRDO. Tendência da mortalidade por doenças respiratórias em idosos no Estado de São Paulo, 1980 a 1998. Rev Saude Publica. 2003;37(2):191-6.

30. Martinez R, Moya MJ. Associação entre paracoccidioidomicose e alcoolismo. Rev Saude Publica. 1992;26(1):12-6.

31. Santos WA, Silva BM, Passos ED, Zandonade E, Falqueto A. Associação entre tabagismo e paracoccidioidomicose: um estudo de casocontrole no Estado do Espírito Santo. Cad Saude Publica. 2003;19(1):245-53.

32. Marques SA, Shikanai-Yasuda MA. Paracoccidioidomycosis associated with immunosuppression, AIDS, and cancer. Em: Franco M, Lacaz CS, Restrepo-Moreno A, Del Negro G, eds. Paracoccidioidomycosis. Boca Raton, Florida: CRC Press; 1993. Pp. 393-405.

33. Conceição YTM. Paracoccidioidomicose e câncer: freqüência da associação em estudo de necrópsias. Rev Soc Bras Med Trop Sao Paulo. 1998;31(6):591-92.

34. Pedro RJ, Aoki FH, Boccato RS, Branchini ML, Gonçalves Junior FL, Papaiordanou PM, et al Paracoccidioidomicose e infecção pelo vírus da imunodeficiência humana. Rev Inst Med Trop Sao Paulo. 1989;31(2):119-25. 
35. Benard G, Duarte AJS. Paracoccidioidomycosis: a model for evaluation of the effects of human immunodeficiency virus infection on the natural history of endemic tropical diseases. Clin Infect Dis. 2000;31(4):1032-9.

36. Nobre V, Braga E, Rayes A, Serufo JC, Godoy $\mathrm{P}$, Nunes N, et al. Opportunistic infections in patients with AIDS admitted to a university hospital of the Southeast of Brazil. Rev Inst Med Trop Sao Paulo. 2003;(45(2):69-74.

37. Santo AH. Avaliação da codificação e do processamento das causas de morte pelo Sistema ACME no Estado de São Paulo, 1992 [tese de livre-docência]. São Paulo: Faculdade de Saúde Pública, Universidade de São Paulo; 1994.

38. Santo AH. Tendência da mortalidade relacionada à varicela no Estado de São Paulo, Brasil, 1985 a 2004: estudo usando causas múlti- plas de morte. Rev Panam Salud Publica. 2007;22(3):132-40.

39. Decreto $\mathrm{N}^{\circ} 6.042$, de 12 de fevereiro de 2007. Altera o Regulamento da Previdência Social, aprovado pelo Decreto ${ }^{\circ} 3.048$, de 6 de maio de 1999, disciplina a aplicação, acompanhamento e avaliação do fator acidentário de prevenção-FAP e do nexo técnico epidemiológico, e dá outras providências. Disponível em: https://www.planalto.gov.br/ccivil_03/_ato 2007-2010/2007/decreto/d6042.htm. Acessado em 3 de agosto de 2007.

40. Ministério da Saúde. Rede Interagencial de Informações para a Saúde. Indicadores e Dados Básicos-Brasil-2006. Indicadores de cobertura. Razão entre óbitos informados e estimados. Disponível em: http://tabnet. datasus.gov.br/cgi/idb2006/f11a.htm. Acessado em 6 de agosto de 2007.
41. Marques SA, Robles AM, Tortorano AM, Tuculet MA, Negroni R, Mendes RP. Mycoses associated with AIDS in the Third World. Med Mycol. 2000;38(suppl 1):269-79.

42. Rozman LM, Santo AH, Rozman MA. Resistência do Mycobacterium tuberculosis às drogas em pacientes HIV+ em cinco municípios da Baixada Santista, São Paulo, Brasil. Cad Saude Publica. 2007;23(5):1051-9.

43. Rozman LM. Tuberculose resistente nos indivíduos HIV positivos em cinco municípios da Baixada Santista-SP [dissertação de mestrado]. São Paulo: Faculdade de Saúde Pública, Universidade de São Paulo; 2004.

Manuscrito recebido em 6 de setembro de 2007. Aceito em versão revisada em 10 de dezembro de 2007.

ABSTRACT Objective. To investigate mortality in which paracoccidioidomycosis appears on any line or part of the death certificate.

Paracoccidioidomycosisrelated mortality trend, state of São Paulo, Brazil: a study using multiple causes of death

Key words
Method. Mortality data for 1985-2005 were obtained from the multiple cause-ofdeath database maintained by the São Paulo State Data Analysis System (SEADE). Standardized mortality coefficients were calculated for paracoccidioidomycosis as the underlying cause-of-death and as an associated cause-of-death, as well as for the total number of times paracoccidioidomycosis was mentioned on the death certificates.

Results. During this 21-year period, there were 1950 deaths related to paracoccidioidomycosis; the disease was the underlying cause-of-death in 1164 cases $(59.69 \%)$ and an associated cause-of-death in 786 (40.31\%). Between 1985 and 2005 records show a $59.8 \%$ decline in the mortality coefficient due to paracoccidioidomycosis as the underlying cause and a 53.0\% decline in the mortality as associated cause. The largest number of deaths occurred among men, in the older age groups, and among rural workers, with an upward trend in winter months. The main causes associated with paracoccidioidomycosis as the underlying cause-of-death were pulmonary fibrosis, chronic lower respiratory tract diseases, and pneumonias. Malignant neoplasms and AIDS were the main underlying causes when paracoccidioidomycosis was an associated cause-of-death. The decision tables had to be adapted for the automated processing of causes of death in death certificates where paracoccidioidomycosis was mentioned.

Conclusions. Using the multiple cause-of-death method together with the traditional underlying cause-of-death approach provides a new angle on research aimed at broadening our understanding of the natural history of paracoccidioidomycosis.

Paracoccidioidomycosis, multiple causes of death, mortality, acquired immunodeficiency syndrome, neoplasms, Brazil. 